\title{
Modified Pocket Endoscopic Submucosal Dissection Technique for Resection of a Giant Rectal Lateral Spreading Tumor
}

\author{
Amol Bapaye ${ }^{1}$ Siddharth Dharamsi ${ }^{1} \quad$ Parag Dashatwar $^{1} \quad$ Guru B. Lakhamaji ${ }^{1}$ \\ ${ }^{1}$ Shivanand Desai Center for Digestive Disorders, \\ Deenanath Mangeshkar Hospital and Research Center, \\ Pune, Maharashtra, India \\ Address for correspondence Amol Bapaye, MD (MS), FASGE, \\ Shivanand Desai Center for Digestive Disorders, Deenanath \\ Mangeshkar Hospital and Research Center, Pune, Maharashtra, \\ 411004, India (e-mail: amolbapaye@gmail.com).
}

J Digest Endosc 2020;10:248-250

Lateral spreading tumors in the rectum (LST's) may be granular or nongranular. Granular LST's (LST-G) can have a nodular component that may harbor intramucosal or invasive cancer. En bloc resection of these lesions is therefore mandatory to provide optimum histological assessment and for the resection to be potentially curative. Endoscopic submucosal dissection (ESD) provides improved curative resection rates and reduced recurrence as compared with piecemeal endoscopic mucosal resection (EMR). ${ }^{1}$ ESD, however, is technically challenging, involves prolonged procedure times and has associated adverse events. ${ }^{2}$ Pocket ESD has been described as a variation to simplify the standard ESD procedure as it maintains submucosal (SM) elevation and tissue lift, provides retraction, and thereby permits quicker dissection.,4

Pocket ESD involves mucosal incision on anal aspect, creation of SM pocket under the lesion, followed by extension and completion of mucosal incision to deliver the specimen. By retaining the peripheral mucosa, fluid leakage is reduced and SM elevation is better maintained. The tip of the endoscope in the SM pocket facilitates tissue traction, facilitating SM dissection in a plane parallel to the muscularis. However, pocket ESD is difficult to perform in very large lesions as the pocket becomes too wide and unwieldy to handle and optimum retraction cannot be maintained. This video case report demonstrates a modification of the standard pocket ESD technique which enabled us to safely resect a large rectal LST.

\section{Description of Video/Procedure}

An 80-year-old male presented with rectal bleeding of 2-month duration. Colonoscopy revealed large hemicircumferential rectal LST-G with large nodular component extending from anal verge up to $12 \mathrm{~cm}$ proximally. Magnification Narrow Band Imaging (NBI) demonstrated a Japan NBI Expert Team (JNET) type IIB pit pattern indicative of an advanced adenoma. En bloc resection was therefore desirable and patient was subjected to a modified pocket ESD technique as demonstrated in the video (Video 1).

Modified pocket ESD was performed using a singlechannel therapeutic gastroscope (1TH GIF-190; Olympus Corporation, Tokyo, Japan) with a transparent distal hood (Olympus) and carbon dioxide insufflation (UCR2 ; Olympus). Distalmost portion of the lesion was elevated by SM injection of gelofusine and diluted adrenaline $(1: 100,000)$ stained with methylene blue. An approximately $2-\mathrm{cm}$ arc shaped mucosal incision was performed on the anal side. SM dissection was performed under the lesion extending to the distal third of the lesion. Dual knife with jet function (Dual-J knife, Olympus) was used for incision and dissection. Diathermy settings on ERBE Vio 200D (ERBE, Tubingen, Germany) were Endocut-Q effect 2, cut duration 3 , cut interval 4 for mucosal incision, and forced coagulation Effect 2, Watts 50 for SM dissection. Once one-third of dissection had been completed, lateral mucosal incision was extended and SM dissection was further performed to deliver the distal third of the lesion. SM pocket was then created in the second third of the lesion using same technique and the procedure was repeated to deliver the second third of the lesion. The final pocket was created underneath the final third of the lesion, mucosal incision was completed, and specimen was delivered after completing dissection. Finally, the proximal third of the lesion ( $\mathbf{- F i g . ~ 1 )}$ ) was completed. The specimen was delivered en bloc and procedure was completed after confirming hemostasis and ruling out deep muscle injury.

Total procedure time was 150 minutes. No intra- or postoperative adverse events were encountered. Final histopathology revealed villous adenoma with focal high-grade dysplasia; no deep invasion and lateral and vertical margins were free of the tumor. Laxatives were prescribed postprocedure, regular diet commenced, and patient was discharged on the subsequent day. Follow-up sigmoidoscopy at 6 weeks 


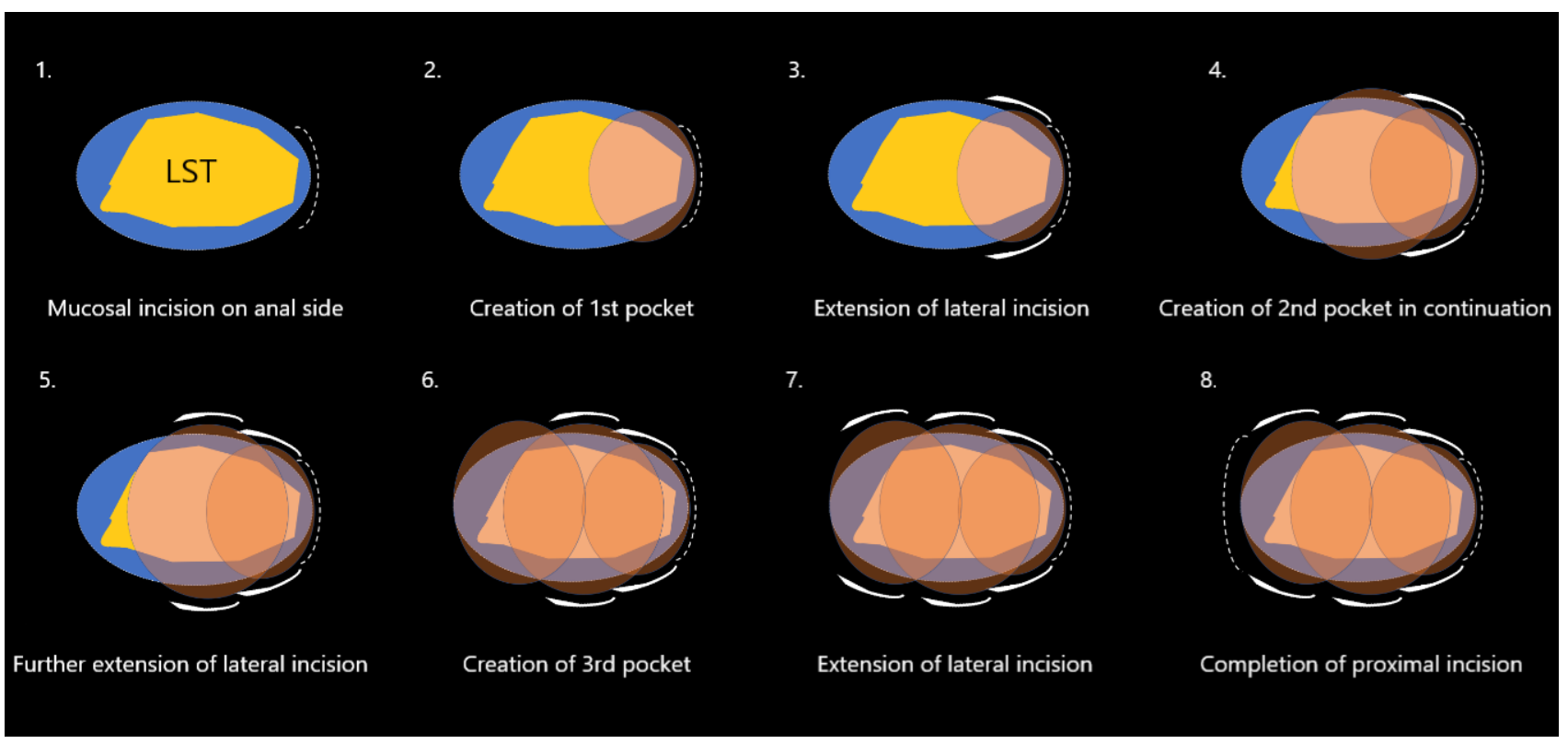

Fig. 1 Cartoon demonstrating the step-by-step technique of modified pocket ESD. ESD, Endoscopic submucosal dissection.

\section{Video 1}

Video demonstrating the step-wise procedure of modified pocketESD.ESD, Endoscopicsubmucosaldissection. Online content including video sequences viewable at: https:// www.thieme-connect.com/products/ejournals/html/ 10.1055/s-0040-1710156.

revealed complete mucosal healing. Follow-up colonoscopy at 6 months revealed healed mucosal scar at ESD site and no recurrence.

\section{Take Home Message}

LST-G's with bulky large nodular component often harbor SM invasion that can be missed on initial assessment using Hi-definition endoscopy. ${ }^{2}$ En bloc ESD is therefore desirable in such cases but can be, however, extremely challenging technically for such a large LST. ${ }^{2}$ Pocket creation ESD technique has been proven to be an efficient technique for ESD in most situations. ${ }^{3,4}$ However, even the pocket ESD can prove challenging in such situations, especially because after a certain depth, the SM pocket becomes very large and wide, cannot provide optimum retraction, and the weight of the partially resected specimen weighs down on the tunnel limiting optimum visualization, advancement of dissection, and thereby effective therapy. It can further also jeopardize procedure safety due to the blind dissection leading to bleeding or perforation.

Using the modified technique, retraction could be better maintained improving the visualization, retraction, and thereby speed, efficiency, and safety of the procedure. By periodically releasing the lateral mucosal attachment, the SM pocket could be reduced to a manageable size, thereby improving dissection efficiency and safety. The decision of creating sequential pockets under one-third of the lesion was an arbitrary estimate by the operator based on the visual appearance. It is the authors' observation that if pocket size increases beyond $4 \mathrm{~cm}$, ESD efficiency drops due to the factors mentioned above. By extending and completing the mucosal incision, this pocket size could be better controlled.

\section{Conclusion}

In conclusion, the authors recommend that the modified pocket ESD technique can be implemented to improve efficiency and safety of ESD for such giant rectal LST's.

\section{Funding}

The authors declare that no funding support was used.

\section{Conflict of Interest}

None declared.

\section{References}

1 Saito Y, Fukuzawa M, Matsuda T, et al. Clinical outcome of endoscopic submucosal dissection versus endoscopic mucosal resection of large colorectal tumors as determined by curative resection. Surg Endosc 2010;24(2):343-352

2 Takeuchi Y, Iishi H, Tanaka S, et al. Factors associated with technical difficulties and adverse events of colorectal endoscopic submucosal dissection: retrospective exploratory factor analysis of a multicenter prospective cohort. Int J Colorectal Dis 2014;29(10):1275-1284

3 Hayashi Y, Miura Y, Yamamoto H. Pocket-creation method for the safe, reliable, and efficient endoscopic submucosal dissection of colorectal lateral spreading tumors. Dig Endosc 2015;27(4):534-535

4 Sakamoto H, Hayashi Y, Miura Y, et al. Pocket-creation method facilitates endoscopic submucosal dissection of colorectal laterally spreading tumors, non-granular type. Endosc Int Open 2017;5(2):E123-E129 